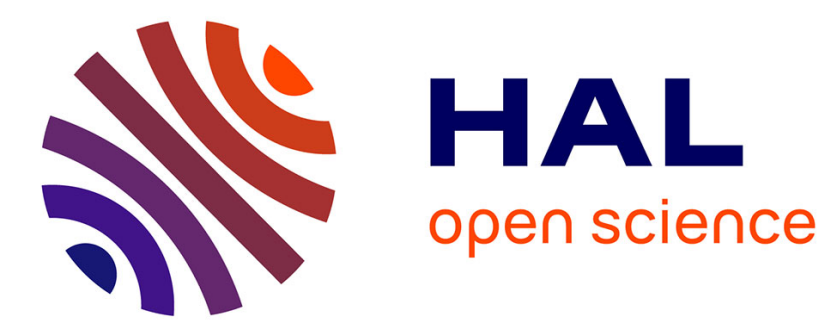

\title{
Lattice Boltzmann BGK simulation of non-linear sound waves: the development of a shock front
}

\author{
Jim Buick, C. Buckley, Clive Greated, Joël Gilbert
}

\section{To cite this version:}

Jim Buick, C. Buckley, Clive Greated, Joël Gilbert. Lattice Boltzmann BGK simulation of non-linear sound waves: the development of a shock front. Journal of Physics A: Mathematical and Theoretical, 2000, 33 (21), pp.3917-3928. 10.1088/0305-4470/33/21/305 . hal-00475067

\section{HAL Id: hal-00475067 https://hal.science/hal-00475067}

Submitted on 21 Apr 2010

HAL is a multi-disciplinary open access archive for the deposit and dissemination of scientific research documents, whether they are published or not. The documents may come from teaching and research institutions in France or abroad, or from public or private research centers.
L'archive ouverte pluridisciplinaire HAL, est destinée au dépôt et à la diffusion de documents scientifiques de niveau recherche, publiés ou non, émanant des établissements d'enseignement et de recherche français ou étrangers, des laboratoires publics ou privés. 


\title{
Lattice Boltzmann BGK simulation of non-linear sound waves: The development of a shock front
}

\author{
J. M. Buick \\ Department of Physics and Astronomy, The University of Edinburgh \\ Mayfield Road, Edinburgh EH9 3.JZ, UK.
}

\section{J. Gilbert}

Laboratoire d'Acoustique de l'Université du Maine

UMR CNRS 6613, Avenue Olivier Messiaen, 72085 Le Mans Cedex 9, FRANCE.

\begin{abstract}
The application of the lattice Boltzmann model to simulating non-linear propagative acoustic waves is considered. The lattice Boltzmann model, and its application to the study on non-linear sound propagation, are discussed. Lattice Boltzmann simulations of the development of a shock front are performed when a sound wave is emitted from a high amplitude sinusoidal source. For a number of parameters, representing different physical situations, the wave development is compared with inviscid shock theory and with the solution of Burgers' equation for a fully viscous fluid. The simulations show good agreement with Burgers' equation and with the inviscid theory when propagation at high Reynolds number is considered. These results suggest that the lattice Boltzmann model is a useful technique for studying a range of problems in non-linear acoustics.
\end{abstract}

PACS numbers: 02.70.-c,43.25.+y

Submitted to: J. Phys. A: Math. Gen.

Electronic mail: J.M.Buick@ed.ac.uk 


\section{Introduction}

The lattice Boltzmann model (LBM) has developed from the lattice gas automata (LGA) $[1,2,3]$ which considers the evolution of a number of idealised fluid 'particles' which move at unit speed on a regular grid subject to particle convection and simplified collision rules which conserve the total fluid mass and momentum. The main application of the LGA has been to fluid dynamics [4, 5, 6], however, sound propagation has also been considered. Frisch et al. [2] showed that the LGA incorporates sound wave propagation in the small perturbation limit. Numerical and theoretical evaluations of this LGA technique were performed by Margolus et al. [7], Chen et al. [8] and Lavallée [9]. The idea was also employed by Chen et al. [10] who proposed a model to directly simulate a linear sound wave without treating the sound wave as a small perturbation limit. The LGA approach to emulating sound waves was also developed by Sudo and Sparrow [11, 12] who considered sound propagation in one and two dimensions and who further developed their model to include dissipation [13]. These developments have lead to a number of successful applications of the LGA to the study of acoustical problems: Numrich et al. [14] considered underwater sound propagation, Stansell and Greated [15] simulated acoustic streaming in a pipe and Rothman [16] and Huang et al. [17] modelled seismic $P$-waves in a homogeneous and inhomogeneous media respectively.

Despite the successful application of the LGA to many problems, both in fluid dynamics and in acoustics, there are a number of difficulties associated with LGA simulations. Two particular problems are the statistical noise associated with the simulation due to the small number of 'particles' being considered, and the viscosity being limited to relatively high values. These both limited the range of application of the LGA, see for example $[9,15]$. In an attempt to overcome these drawbacks the LGA evolved in a number of stages and developed into the LBM; details of the various steps can be found in, for example $[18,19]$. The development of the LBM was driven mainly for its use in fluid dynamics since the LBM can be shown [18] to mimic the incompressible NavierStokes and continuity equations. Recently Buick et al. [20] have applied the technique to simulate linear sound waves when the pressure variations are considered to be a small perturbation. Here we extend the scope of the simulations to consider non-linear waves and show that the LBM method and the incompressible approximation are not limited to the linear regime. In all the simulations the amplitude of the density variation is no greater than $1 \%$ of the ambient density so that the incompressible LBM equations can be applied to a good approximation [20].

\section{The Lattice Boltzmann Model}

The LBM considered here evolves on a fixed hexagonal lattice. The sites on the lattice are joined by unit vectors $\boldsymbol{e}_{i}$, where $i=1,2, \ldots, 6$, while $\boldsymbol{e}_{0}$ is defined to be the null 
Figure 1. The hexagonal grid on which the simulations are performed. The vectors $\boldsymbol{e}_{1}, \ldots, \boldsymbol{e}_{6}$ are unit vectors along the directions of the grid and $\boldsymbol{e}_{0}$ is the null vector.

vector; see figure 1. The simplified, discretised Boltzmann equation [21, 22] is given by

$$
f_{i}\left(\boldsymbol{r}+\boldsymbol{e}_{i}, t+\Delta t\right)-f_{i}(\boldsymbol{r}, t)=\Omega_{i}(\boldsymbol{r}, t) \quad i=0,1, \ldots, 6,
$$

where $f_{i}(\boldsymbol{r}, t)$ are the distribution functions along the links $\boldsymbol{e}_{i}$ at site $\boldsymbol{r}$ and time $t$ and $\Omega_{i}(\boldsymbol{r}, t)$ is the collision operator. The left hand side of equation (1) is the convection operator and describes streaming of the distribution functions on the grid. This operator can be seen to be linear in velocity space. The simplification of the Boltzmann equation occurs in the form of the collision operator which is taken to be the BGK approximation $[23,21,24]$ :

$$
\Omega_{i}(\boldsymbol{r}, t)=-\frac{1}{\tau}\left[f_{i}(\boldsymbol{r}, t)-\bar{f}_{i}(\boldsymbol{r}, t)\right]
$$

where $\bar{f}_{i}$ is the equilibrium distribution function and $\tau$ is the relaxation time. The form of $\Omega_{i}$, given in equation (2), represents a relaxation of the distribution towards its equilibrium value and recovers the non-linear form of the fluid, ensuring that the fully non-linear Navier-Stokes equation is satisfied. The equilibrium distribution functions depend only on the fluid density, $\rho$, and velocity, $\boldsymbol{u}$, at each site which can be calculated from the distribution functions as

$$
\rho=\sum_{i} f_{i}
$$

and

$$
\rho u_{\alpha}=\sum_{i} f_{i} e_{i \alpha}
$$

where the Greek subscripts represent vector components and summation over repeated Greek indices is assumed. Thus the collision process requires only local information to introduce non-linear effects into the simulation. Up to $O\left(u^{2}\right)$, we assume that the equilibrium distribution function has the general form [25],

$$
\bar{f}_{i}(\boldsymbol{r}, t)= \begin{cases}\rho\left(A+B \boldsymbol{e}_{i} \cdot \boldsymbol{u}+C\left(\boldsymbol{e}_{i} \cdot \boldsymbol{u}\right)^{2}+D u^{2}\right), & i=1, . ., 6 \\ \rho\left(A_{0}+D_{0} u^{2}\right) & i=0 .\end{cases}
$$

This expansion up to second order in $\boldsymbol{u}$ ensures that the simulations have second order accuracy, however the expansion is only valid for small Mach numbers $M=u / c_{s}$, where $c_{s}$ is the speed of sound in the medium. The constants $A, A_{0}, \ldots, D_{0}$ can be found for the specific lattice being used and the required properties of the fluid. Here we require that the collisions conserve mass and momentum, that is

$$
\rho=\sum_{i} \bar{f}_{i}
$$

and

$$
\rho u_{\alpha}=\sum_{i} \bar{f}_{i} e_{i \alpha}
$$


and that the fluid is isotropic and exhibits Galilean invariance. These requirements are fulfilled by

$$
A=\frac{1-d_{0}}{6}, \quad A_{0}=d_{0}, \quad B=\frac{1}{3}, \quad C=\frac{2}{3}, \quad D=\frac{-1}{6} \quad \text { and } \quad D_{0}=-1,(8)
$$

where $d_{0}$ is an arbitrary constant.

The macroscopic equations can be derived from the lattice Boltzmann equations by performing a multiscaling Chapman-Enskog expansion [2] in the time and space derivatives such that

$$
\frac{\partial}{\partial t} \rightarrow \epsilon \frac{\partial}{\partial t_{1}}+\epsilon^{2} \frac{\partial}{\partial t_{2}}
$$

and

$$
\frac{\partial}{\partial x} \rightarrow \epsilon \frac{\partial}{\partial x_{1}}
$$

and the distribution function is expanded about the equilibrium value,

$$
f_{i} \rightarrow \bar{f}_{i}+\epsilon f_{i}^{(1)}+\epsilon^{2} f_{i}^{(2)},
$$

where $\epsilon$ is the Knudsen number which must be small. If we further assume that the lattice spacing, $\boldsymbol{e}_{i}$, and the time-step, $\Delta t$, are small parameters, and of the same order as $\epsilon$, then equation (1) can be Taylor expanded and combined with equation (2). Substituting the expression for the equilibrium distribution functions, equation (5), and the Chapman-Enskog expansions, equations (9)-(11), and retaining terms up to second order in $\epsilon$ we obtain [25] the continuity and Navier-Stokes equations for an incompressible, isothermal fluid in two-dimensions:

$$
\partial_{t} \rho+\partial_{\alpha} \rho u_{\alpha}=0
$$

and

$$
\partial_{t} \rho u_{\alpha}+\partial_{\beta} \rho u_{\beta} u_{\alpha}=-\partial_{\alpha}\left(\frac{1-d_{0}}{2}\right) \rho+\nu \partial_{\beta} \partial_{\beta} \rho u_{\alpha}+\zeta \partial_{\alpha} \partial_{\beta} \rho u_{\beta},
$$

where

$$
\nu=\frac{\tau-1 / 2}{4}
$$

and

$$
\zeta=\left(\tau-\frac{1}{2}\right)\left[\frac{1}{2}-\frac{\left(1-d_{0}\right)}{2}\right]
$$

are the kinematic shear and bulk viscosities. The pressure term in equation (13) is $p=\left(1-d_{0}\right) \rho / 2$ which, for a perfect gas, gives the speed of sound as $c_{s}=\left[\left(1-d_{0}\right) / 2\right]^{1 / 2}$.

In deriving equations (12) and (13) there have been a number of assumptions made which restrict the application of the LBM. In the expansion of the equilibrium distribution function it is assumed that the Mach number $M=u / c_{s}$ is small; in the Taylor expansion of the Boltzmann equation the length and time scales of the simulation, 
$\boldsymbol{e}_{i}$ and $\Delta t$, are assumed to be small; finally the density variation must also be assumed to be small since the equations of motion are for an incompressible fluid. Since we are dealing with a perfect gas where $p$ is proportional to $\rho$ we can write, for a progressive plane wave,

$$
\frac{\rho^{\prime}}{\rho_{0}}=\frac{u}{c_{s}}=M
$$

where $\rho^{\prime}$ is a density variation due to the sound wave and $\rho_{0}$ is the ambient density. Here the low Mach number approximation and the incompressibility condition reduce to the one constraint. The further constraint that $\boldsymbol{e}_{i}$ and $\Delta t$ are small requires that the macroscopic scales of the wave are much larger than the microscopic scales of the grid, that is for a wave of wavelength $\lambda$ and period $T$

$$
\left|\boldsymbol{e}_{i}\right| \ll \lambda \text { and } \Delta t \ll T \text {. }
$$

This can always be achieved by selecting suitable values of $\lambda$ and $T$.

It is worth noting that while the LBM satisfies the incompressible Navier-Stokes equation the simulated fluid can experience density variations. These arise from the definition of $\rho$, equation (3), which does not constrain $\rho$ to be constant. In many fluid dynamics applications, for example pressure driven Poiseuille flow, this is seen as a disadvantage since so called compressibility errors are observed in a LBM simulation [26] and in many cases the pressure gradient is approximated by a body force to remove this effect [27]. Here we use this feature of the LBM in the limit that we consider only low Mach numbers.

\section{Non-linear Acoustics}

In this section we consider acoustic waves where the amplitude is large enough that nonlinear motion is observed, but where the restriction that the Mach number, $M=u / c_{s}$, is small, as is required for the lattice Boltzmann model to be applicable. Under these conditions non-linear phenomena are locally small (of the order of $M$ ), however the effects are cumulative and increase with the distance of propagation and will, after a sufficient propagation distance, significantly distort the wave. To consider this we start from the Navier-Stokes and continuity equations and the equation of state of the fluid, see for example [28, 29, 30]:

$$
\begin{aligned}
& \partial_{t} \rho+\partial_{\alpha} \rho u_{\alpha}=0 \\
& \rho\left[\partial_{t} u_{\alpha}+u_{\alpha} \partial_{\beta} u_{\beta}\right]=-\partial_{\alpha} p+\rho \nu \partial_{\beta} \partial_{\beta} u_{\alpha}+\rho\left[\zeta+\nu\left(1-\frac{2}{D}\right)\right] \partial_{\alpha} \partial_{\beta} u_{\beta},
\end{aligned}
$$

and

$$
p^{\prime}=c_{s}^{2} \rho^{\prime}+\frac{(\gamma-1) c_{s}^{2}}{2 \rho_{0}} \rho^{2}-\chi\left(\frac{1}{c_{v}}-\frac{1}{c_{p}}\right) \partial_{\alpha} u_{\alpha},
$$

where $D$ is the number of dimensions, $\chi$ is the coefficient of thermal conductivity, $c_{p}$ and $c_{v}$ are the specific heats at constant pressure and volume, $\gamma=c_{p} / c_{v}$ and the pressure and 
density ( $p$ and $\rho$ ) are respectively defined as the sum of the ambient ( $p_{0}$ and $\rho_{0}$ ) and the perturbation $\left(p^{\prime}\right.$ and $\rho^{\prime}$ ). The kinematic shear and bulk viscosities and the speed of sound are $\nu, \zeta$ and $c_{s}$ respectively, as before. Substituting the equation of state, equation (20), into the continuity and Navier-Stokes equations (18) and (19), expressing the pressure and the density as the sum of the ambient value and the perturbation, and neglecting the term $\rho^{\prime} u_{\beta} \partial_{\beta} u_{\alpha}$ since it is $O\left(M^{3}\right)$ we obtain

$$
\frac{\partial \rho^{\prime}}{\partial t}+\left(\rho_{0}+\rho^{\prime}\right) \frac{\partial u}{\partial x}+u \frac{\partial \rho^{\prime}}{\partial x}=0
$$

and

$$
\left(\rho_{0}+\rho^{\prime}\right) \frac{\partial u}{\partial t}+\rho_{0} u \frac{\partial u}{\partial x}=-c_{s}^{2} \frac{\partial \rho^{\prime}}{\partial x}-\frac{2 \epsilon c_{s}^{2} \rho^{\prime}}{\rho_{0}} \frac{\partial \rho^{\prime}}{\partial x}+\rho_{0} b \frac{\partial^{2} u}{\partial x^{2}},
$$

where $b=\zeta+(1+1-2 / D) \nu+\chi\left(1 / c_{v}-1 / c_{p}\right) / \rho_{0}, \epsilon=(\gamma+1) / 2$ and we have used one-dimensional notation since we are interested in the propagation of plane waves.

To proceed further it is not possible to consider a classical perturbation expansion of equations (21) and (22) using linear wave theory as a first order approximation. This is because linear theory does not give a satisfactory first approximation, since no matter how small the initial amplitude the long-term behaviour will be non-linear in the absence of dissipation. It is therefore necessary to use a multiple scale method where $x, t$ and $X=M x$ are considered to be independent variables and $\partial / \partial x \rightarrow \partial / \partial x+M \partial / \partial X$. This allows a good description of the wave up to distances $x=O(1 / M)$. Following this approach and introducing $\tau=t-x / c_{s}$, equations (21) and (22) can be shown to satisfy Burgers' equation [28]

$$
\frac{\partial u}{\partial x}-\frac{\epsilon}{c_{s}^{2}} u \frac{\partial u}{\partial \tau}=\frac{b}{2 c_{s}^{3}} \frac{\partial^{2} u}{\partial \tau^{2}} .
$$

Finally, it is convenient to change to dimensionless variables $q=u / U, \sigma=\epsilon M k x$ and $\theta=\omega \tau$ where $U, \omega$ and $k$ are the initial velocity, angular frequency and wave number of the source and $M$ has been re-defined here as $M=U / c_{s}$. In these co-ordinates Burgers' equation is

$$
\frac{\partial q}{\partial \sigma}-q \frac{\partial q}{\partial \theta}=\kappa \frac{\partial^{2} q}{\partial \theta^{2}}
$$

where

$$
\kappa=\frac{1}{2 \epsilon} \frac{1}{\operatorname{Re}} \frac{1}{M}
$$

and Re is the acoustical Reynolds number given by

$$
\operatorname{Re}=\frac{c_{s}}{b k} .
$$

The dimensionless propagation parameter, $\sigma$, describes the development of the shock wave for $0 \leq \sigma \leq 1$. The shock formation distance for a sinusoidal sound wave with amplitude unity is $\sigma=1$. That is, $\sigma=1$ determines the distance at which a sinusoidal wave in an inviscid fluid is transformed into a discontinuous wave of sawtooth shape. In a viscous fluid the wave is transformed into an almost sawtooth shape, however, 
the action of viscous damping prevents a total discontinuity forming. Note that the multiple scale method used in the derivation of Burgers' equation is valid for distances up to $x=O(1 / M)$, that is distances of the order of the shock formation distance $\sigma=1$.

\subsection{Solution of Burgers' equation}

Here we consider the solution of Burgers' equation for two cases, firstly the analytic solution for the special case for an inviscid medium where $\kappa=0$, and secondly the numerical solution of the full Burgers' equation.

3.1.1. Inviscid Fluid We wish to solve the inviscid Burgers' equation

$$
\frac{\partial q}{\partial \sigma}-q \frac{\partial q}{\partial \theta}=0
$$

for the initial condition

$$
\left.q(\sigma, \theta)\right|_{\sigma=0}=f(\theta) .
$$

Following Crighton et al. [29] we consider a curve in the $(\sigma, \theta)$ plane for which $\theta$ is some definite function of $\sigma$. On this curve we have

$$
\frac{\mathrm{d} q}{\mathrm{~d} \sigma}=\frac{\partial q}{\partial \sigma}+\left(\frac{\mathrm{d} \theta}{\mathrm{d} \sigma}\right) \frac{\partial q}{\partial \theta}=0 \quad \text { if } \quad \frac{\mathrm{d} \theta}{\mathrm{d} \sigma}=-q .
$$

Let $\Gamma$ be one such curve where $q$ is constant, then $\Gamma$ is the straight line $\theta=-q \sigma+\phi$. The point where this line cuts the $\theta$-axis occurs when $\sigma=0$, which gives $\phi=\theta$ or

$$
q=f(\phi),
$$

where $\phi$ is defined through

$$
\phi=\theta+q \sigma .
$$

Now, equation (27) preserves the parity and periodicity of the initial function. Thus, if we now consider the special case of a sinusoidal source, $f(\theta)=\sin \theta$, the solution of equation (27) must have the form

$$
q(\sigma, \theta)=\sum_{n=1}^{\infty} a_{n}(\sigma) \sin (n \theta)
$$

where

$$
a_{n}(\sigma)=\frac{2}{\pi} \int_{0}^{\pi} q(\sigma, \theta) \sin n \theta \mathrm{d} \theta
$$

Substituting in equations (30) and (31) and changing the variable of integration from $\theta$ to $\phi$, equation (33) can be expressed as

$$
a_{n}(\sigma)=\frac{2}{n \sigma} J_{n}(n \sigma)
$$

where $J_{n}$ is the Bessel function of order $n$. This gives the Fubini-Ghiron solution for the development of a shock in an inviscid fluid:

$$
q=\sum_{n=1}^{\infty} \frac{2}{n \sigma} J_{n}(n \sigma) \sin (n \theta) .
$$

This solution holds for $0 \leq \sigma \leq 1$ since for $\sigma>1$ the $\theta-\phi$ relationship in not $1-1$ and the change of integration variable in not possible. 
3.1.2. Numerical Solution We now turn our attention to the numerical solution of equation (24) [31]. To do this we consider a travelling wave of the form

$$
q=\sum_{n=1}^{\infty} a_{n}(\sigma) \sin n \theta
$$

Considering first the non-linear term:

$$
q \frac{\partial q}{\partial \theta}=\left[\sum_{n=1}^{\infty} a_{n}(\sigma) \sin n \theta\right]\left[\sum_{m=1}^{\infty} m b_{m}(\sigma) \cos m \theta\right] .
$$

Collecting together terms with the same frequency this can be re-written as

$$
q \frac{\partial q}{\partial \theta}=\frac{1}{2} \sum_{m=1}^{\infty}\left[m \sin m \theta\left(\sum_{p=1}^{m-1} \frac{a_{p} a_{m-p}}{2}-\sum_{p=m+1}^{\infty} a_{p-m} a_{p}\right)\right] .
$$

Calculating the other derivatives the solution of Burgers' equation reduces to solving the following set of first-order partial differential equations

$$
\frac{\partial a_{n}}{\partial \sigma}=n\left(\sum_{p=1}^{n-1} \frac{a_{p} a_{n-p}}{2}-\sum_{p=n+1}^{\infty} a_{p-n} a_{p}\right)-\kappa n^{2} a_{n},
$$

where $\left.a_{1}\right|_{\sigma=0}=1$ and $\left.a_{n}\right|_{\sigma=0}=0$ for $n \geq 2$. This can be solved by truncating the series to $N$ harmonics and solving the $N$ equations using a variable-order variable-step Adams method $[32,33]$. The solutions presented here were truncated at $N=20$, although only the first six harmonics are plotted.

\section{Numerical Simulations}

The development of the shock wave was simulated using the lattice Boltzmann model described in section 2. This was done using a grid consisting of $\lambda$ sites in the $x$-direction and $m$ sites in the $y$-direction, where $\lambda$ is the wavelength of the sound wave being simulated and $m$ is an arbitrary number. Periodic boundary conditions were applied at each of the grid edges. Since plane waves are being simulated the value of the pressure and the velocity, and hence the distribution functions, $f_{i}$, are the same on each column ( $x$ constant) and so the value of $m$ is totally arbitrary. Here $m=4$ was used. A sinusoidal source was mimicked by initialising the grid with a sinusoidal pressure (density) and velocity variation. That is, the velocity and density were specified according to

$$
\rho=\rho_{0}+a \sin \left(\frac{2 \pi x}{\lambda}\right)
$$

and

$$
u=\frac{a c_{s}}{\rho_{0}} \sin \left(\frac{2 \pi x}{\lambda}\right)
$$

The initial values of $f_{i}$ at $t=0$ were then calculated by substituting these values into equation (5). The constant $d_{0}$ in equation (8) can in general be varied to change the ratio of the shear and bulk viscosities; here is was fixed at $d_{0}=1 / 2$. The value of the amplitude parameter $a$ in equations (40) and (41) and the ambient density $\rho_{0}$ determine the Mach number: $M=a / \rho_{0}$. Here we choose $a=0.1$ and $\rho_{0}=10$ giving $M=0.01$ 
Figure 2. The measured pressure variation as a function of time for the first 16 periods of oscillation. This corresponds to the development of the shock front.

Figure 3. A stacked profile of the pressure variation as a function of the wave phase at selected times during the development of the shock front. The distortion of the initial sinusoidal variation to the ' $N$ '-shaped shock wave is clearly visible.

which satisfies the lattice Boltzmann constraint that $M \ll 1$. It is usual to express the intensity of a sound wave in terms of the sound pressure level rather than the Mach number. Comparing the simulations to a sound wave in air at atmospheric pressure, $M=0.01$ corresponds to a pressure variation of $1 \times 10^{3} \mathrm{~Pa}$ which gives a sound pressure level [20] of $20 \log \left(1 \times 10^{3} / 2 \times 10^{-5}\right)=154 \mathrm{~dB}$ SPL. The simulation is then allowed to evolve and the density and velocity measured at position $x=\lambda / 2$ giving a time series record of the wave. The lattice exhibits periodicity and hence all other positions, for a particular phase, are equivalent. This differs from a typical experimental setup and the theory in section 3 where a source is positioned in a medium and measurements are made at different positions giving a spatial record of the sound wave. Complete information for a particular phase can be obtained by measurements at different distances from the source at a single arbitrary time. The constant dispersion relation of our simulation ensures complete space-time ergodicity and these two situations become completely equivalent. That is to say, measurements at time $t$ and $t+\delta t$ from our simulations can equally be thought of as measurements at positions $x$ and $x+\delta x$ where $x=c_{s} t$ and $\delta x=c_{s} \delta t$.

The LBM described in section 2 has equation of state $p=c_{s}^{2} \rho$ which corresponds to a fluid with $\gamma=1$, with no thermal energy dissipation. This arises from the term $\partial_{\alpha}\left(1-d_{0}\right) \rho / 2$ in equation (13) being equated to the term $\partial_{\alpha} p$ in the Navier-Stokes equation, with $c_{s}=\left[\left(1-d_{0}\right) / 2\right]^{1 / 2}$. We note that a different choice for the equilibrium distribution function, equation (5), could change the equation of state of the simulated fluid [34]. The dissipative term $b$ is now expressed as $b=\nu+\zeta$ (since we are considering two-dimensions) and the constant $\epsilon=1$. Thermal dissipation effects can, however, be simulated using an effective viscosity which incorporates both viscous and thermal losses. This was not done here.

\section{Simulation Results}

A typical set of results is shown in figure 2 which shows the variation in the normalised pressure with time during the formation of the shock wave. Initially the variation is approximately sinusoidal, however this can be see to change as the wave evolves. This distortion of the initial sine wave can be seen more clearly in figure 3 which shows a stack profile representing the normalised pressure of the wave plotted against the wave phase at different times. The change in the form of the oscillation can be clearly seen 
Figure 4. A typical example of the Fourier transform of a three wavelength segment of the signal at selected times during the evolution of the shock wave. (a) represents the initial segment at $x=3 \lambda / 2$ and shows most of the wave energy concentrated in the fundamental harmonic with the amplitude of the second and third harmonic starting to increase. (b) and (c) show the spectrum at later times and show the increasing prominence of the higher harmonics and the decrease of the fundamental harmonic as $\sigma$ increases.

Figure 5. The variation in the relative amplitude of the first six harmonics for an initially sinusoidal non-linear wave during the development of the shock front for three different Reynolds numbers, the Mach number is $M=0.01$. In each case the numerical solution of Burgers' equation (24) is represented by a solid line. Also shown for comparison is the Fubini-Ghiron solution, equation (35), for each of the harmonics.

in figure 3.

In order to compare these results with the theoretical analysis we need to look at the growth and decay of the fundamental and higher harmonics within the waves. This was done by dividing the results into segments with length $3 T$ in such a way that segment $l$ contains $(l-1) T+1<t<(l+2) T$. Each of these segments was then Fourier transformed and the resulting spectrum considered to represent the wave at $x=(l+1 / 2) \lambda$. Typical results of such a procedure are shown in figure 4 which shows the change in the spectrum at selected distances from the source. As expected the amplitude of the higher harmonics is seen to increase with propagation distance, this is due to energy being transferred to these harmonics from the fundamental harmonic which is seen to decrease in amplitude. The change in the magnitude of each frequency component was extracted from the Fourier transform for waves with three different Reynolds numbers, $R e \simeq 1,600,1,100$ and 160 . This was achieved using a fixed wavelength $\lambda=500$ and varying the fluid viscosity using $\tau=0.55,0.57$ and 0.95 respectively. In each case the Mach number was fixed at $M=0.01$. These are shown in figure 5 for the first six harmonics along with the numerical solution of Burgers' equation and the inviscid Fubini-Ghiron solution for comparison. In general the simulation results show excellent agreement with the numerical solution of Burgers' equation. There are, however, some regions where there is a small deviation. One source of error in the analysis is that we are performing the Fourier transform over three wavelengths during which there is a change in the amplitudes of the harmonics, both due to energy transfer between the harmonics and due to viscous damping. Therefore the Fourier transform will not only contain peaks at the frequency of the harmonics, but also contributions due to the change in these amplitudes over the sample. This can be seen in figures 4 (a) and (b) where the values between $n=1$ and $n=2$ and between $n=2$ and $n=3$ are not zero. These figures represent the early stages in the development of the shock wave where there is the largest change in the amplitude of the harmonics. At later times, see figure 4 (c), the change in the harmonic amplitudes is smaller and so is the value of the 
Fourier transform between the harmonics. In general these additional contributions are small and, combined with numerical error, account for the small deviations observed, particularly when the harmonic amplitude is small; see for example figure 5 (d)-(f) at small $\sigma$. As expected the results approach the inviscid Fubini-Ghiron solution as the Reynolds number increases.

\section{Conclusion}

The use of a BGK lattice Boltzmann model for simulating non-linear propagative acoustic waves has been considered. It has been seen that a range of problems in non-linear acoustics are within the dynamic range of the lattice Boltzmann model and the application of the technique has been demonstrated. This was done by simulating the development of a shock front from an initially sinusoidal non-linear wave. The results of the simulation agreed well with theory, suggesting that the lattice Boltzmann model is indeed a useful approach to simulating non-linear acoustical phenomena.

The simulations presented here have been limited to considering progressive waves in an unbound media. This is not a fundamental restriction of the technique which should be equally applicable to studying standing waves and propagation in a pipe where the action of the walls significantly influences the acoustics; indeed the ability of the LBM to model complex boundary situations is well established. An investigation of this and the steady state acoustic streaming flows setup by the attenuation of a sound field in the boundary layer is currently in progress.

\section{Acknowledgments}

This work was partially supported by EPSRC, UK.

\section{References}

[1] U. Frisch, B. Hasslacher and Y. Pomeau. Phys. Rev. Lett. 5615051986.

[2] U. Frisch, D. d' Humières, B. Hasslacher, P. Lallemand, Y. Pomeau and J.-P. Rivet. Complex Systems 16491987.

[3] S. Wolfram. J. Stat. Phys. 454711986.

[4] Proceedings of Workshop of Large Nonlinear Systems, Santa Fe, edited by G. D. Doolen (1986). Published in Complex Systems 15451987.

[5] Proceedings of Lattice Gas Methods for PDEs: Theory, Application and Hardware, Los Almos, edited by G. D. Doolen (1989). Published in Physica D 4711991.

[6] Proceedings of Advanced Research Workshop on Lattice Gas Automata, Nice, edited by J.-P. Boon, 1991. Published in J. Stat. Phys. 683471992.

[7] N. Margolus, T. Toffoli and G. Vichniac. Phys. Rev. Lett. 5616941986.

[8] H. Chen, S. Chen and G. D. Doolen. Phys. Lett. A 1631611989.

[9] P. Lavallée. Phys. Lett. A 1633921992.

[10] H. Chen, S. Chen, G. D. Doolen and Y. C. Lee. Complex Systems 22591988. 
[11] Y. Sudo and V. W. Sparrow. J. Comput. Acoust. 14231993.

[12] Y. Sudo and V. W. Sparrow. AIAA J. 3315821995.

[13] Y. Sudo and V. W. Sparrow, J. Comput. Acoust. 3691995.

[14] S. K. Numrich, R. A. Krutar and R. Squier. In Computational Acoustics Volume 1, North-Holland 811993.

[15] P. Stansell and C. A. Greated. Phys. Fluids 932881997.

[16] D. H. Rothman. Geophys. Res. Lett. 14171987.

[17] J. Huang, Y. Chu and C. Yin. Geophys. Res. Lett. 1512391988.

[18] S. Chen and G. D. Doolen. Annu. Rev. Fluid Mech. 303291998.

[19] R. Benzi, S. Succi and M. Vergassola. Phys. Rep. 2221461992.

[20] J. M. Buick, C. A. Greated and D. M. Campbell. Europhys. Lett. 432351998.

[21] S. Chen, H. Chen, D. Martinez and W. Matthaeus. Phys. Rev. Lett. 6737761991.

[22] Y. H. Qian, D. d' Humières and P Lallemand. Europhys. Lett. 174791992.

[23] P. L. Bhatnagar, E. P. Gross and M. Krook. Phys. Rev. 945111954.

[24] S. Chen, Z. Wang, X. Shan and G. D. Doolen. J. Stat. Phys. 683791992.

[25] H. Chen, S. Chen and H. Matthaeus. Phys. Rev. A 4553391992.

[26] Z. Lin, H. Fang and R. Tao. Phys. Rev. E 5463231996.

[27] Q. Zou, S. Hou and G. D. Doolen. J. Stat. Phys. 813191995.

[28] O. V. Rudenko and S. I. Soluyan. Theoretical Foundations of Nonlinear Acoustics. Consultants Bureau, New York 1977.

[29] D. G. Crighton, A. P. Dowling, J. E. Ffowcs Williams, M. A. Heckl and F. A. Leppington. Modern Methods in Analytical Acoustics, Springer-Verlag 1992.

[30] L. Landau and E. Lifshitz. Fluid Mechanics, Pergamon Press, New York 1963.

[31] L. Menguy and J. Gilbert. Submitted to Acustica - Acta Acustica 1999.

[32] G. Hall and J. M. Watt. Modern Numerical Methods for Ordinary Differential Equations, Clarendon Press, Oxford 1976.

[33] Nag Routine D02CFJ, The NAG Fortran Library, Mark 141990.

[34] D. H. Rothman and S. Zaleski Lattice-Gas Cellular Automata. Simple models of complex hydrodynamics. Cambridge University Press, Cambridge 1997. 